\title{
On Distance Function in Some New Analytic Bergman Type Spaces in $\mathbb{C}^{n}$
}

\author{
Romi F. Shamoyan ${ }^{1}$ and Olivera R. Mihić ${ }^{2}$ \\ ${ }^{1}$ Bryansk University, 241050 Bryansk, Russia \\ ${ }^{2}$ Fakultet Organizacionih Nauka, Jove Ilića 154, 1100 Belgrade, Serbia \\ Correspondence should be addressed to Olivera R. Mihić; oliveradj@fon.rs
}

Received 2 February 2014; Accepted 23 March 2014; Published 6 May 2014

Academic Editor: Kehe Zhu

Copyright (c) 2014 R. F. Shamoyan and O. R. Mihić. This is an open access article distributed under the Creative Commons Attribution License, which permits unrestricted use, distribution, and reproduction in any medium, provided the original work is properly cited.

\begin{abstract}
We extend our previous sharp results on distances obtained for analytic Bergman type spaces in unit disk to some new analytic Bergman type spaces in higher dimensions in $\mathbb{C}^{n}$. Also, we study the same problem in anisotropic mixed norm $h(p, q, s)$ spaces consisting of $n$-harmonic functions on the unit polydisc of $\mathbb{C}^{n}$.
\end{abstract}

\section{Introduction and Preliminaries}

The goal of this paper is to add several new results for distances in analytic Bergman type and Bergman type nharmonic function spaces of functions of several variables.

It turns out that some of our distance theorem, which we proved before for the case of unit disk, are valid under certain natural conditions in various domains and various Bergman type analytic spaces. Namely, we look at analytic Bergman spaces in minimal ball and so-called analytic $K$ Bergman spaces and in analytic Bergman-Orlicz spaces. These analytic spaces act as direct extensions of well-known analytic Bergman spaces in the unit disk. These analytic spaces are relatively new and we will include some basic facts on them in our paper; they will also be needed for proofs of our assertions partially. We will turn also to polydisk and $n$-harmonic function spaces on them providing also a sharp result there. Note in less general case, namely, in analytic Bergman type spaces in polydisk, this result is already known (see $[1,2]$ ).

Our intention in this paper is the same as our previous papers on this topic; namely, we collect some facts from earlier investigation concerning Bergman projection and Bergman kernel and use them for our purposes in estimates of $\operatorname{dist}_{Y}(f, \mathscr{X})$ function (distance function).
Following our previous paper $[1,2]$, we easily see that, to obtain a sharp result for distance function, we only need several tools and the scheme here is as follows. First we need an embedding of our quazinormed analytic space (in any domain) into another one $(X \subset Y)$, this immediately poses a problem of $\operatorname{dist}_{Y}(f, X)=\inf _{g \in X}\|f-g\|_{Y}$ for all $f \in Y \backslash X$, and then we need the Bergman reproducing formula for all $f$ functions from $Y$ space. Then, finally we use the boundedness of Bergman type projections, with $|K(z, w)|$ positive kernel acting from $X$ to $X$, together with Forelli-Rudin type sharp estimates of Bergman kernel. These three tools were used in general Siegel domain of second type, polydisk, and unit ball in [1-4] (see also various references there). We continue to use these tools providing new sharp (and not sharp) results in various spaces of analytic functions in this paper.

First we provide a known result in the unit disk with complete proof taken from our previous paper $([1,2])$. In the unit disk case all arguments here are short and transparent and are based on several tools like Forelli-Rudin type estimate and estimates for Bergman type projections with positive Bergman kernel, and then we will see arguing similarly as in unit disk; we will complete the proof of more complicated cases easily. The complete formulation of our theorems will be given, but only sketches of proofs will be added and details of proofs of higher dimensional cases will be left to readers. 
Note that it is easy to see that our assertions may have various applications in approximation theory, for example.

We will need various definitions for formulations of main results. All facts about polydisk are taken from [5].

Let $n \in \mathbb{N}$ and $\mathbb{C}^{n}=\left\{z=\left(z_{1}, \ldots, z_{n}\right): z_{k} \in \mathbb{C}, 1 \leq k \leq\right.$ $n$ \} be the $n$-dimensional space of complex coordinates. We denote the unit polydisk by

$$
\mathbf{U}^{n}=\left\{z \in \mathbb{C}^{n}:\left|z_{k}\right|<1,1 \leq k \leq n\right\}
$$

and the distinguished boundary of $\mathbf{U}^{n}$ by

$$
T^{n}=\left\{z \in \mathbb{C}^{n}:\left|z_{k}\right|=1,1 \leq k \leq n\right\} .
$$

By $m_{2 n}$ we denote the volume measure on $\mathbf{U}^{n}$ and by $m_{n}$ we denote the normalized Lebesgue measure on $T^{n}$. Let $H\left(\mathbf{U}^{n}\right)$ be the space of all holomorphic functions on $\mathbf{U}^{n}$.

Throughout the paper, we write $C$ (sometimes with indexes) to denote a positive constant which might be different at each occurrence (even in a chain of inequalities) but is independent of the functions or variables being discussed.

The notation $A \asymp B$ means that there is a positive constant $C$, such that $B / C \leq A \leq C B$. We will write for two expressions $A \lesssim B$ if there is a positive constant $C$ such that $A<C B$. $I^{n}$.

We denote the interval $(0,1)$ by $I$, accordingly $(0,1)^{n}$ by

The Hardy spaces, denoted by $H^{p}\left(\mathbf{U}^{n}\right)(0<p \leq \infty)$, are defined by $H^{p}\left(\mathbf{U}^{n}\right)=\left\{f \in H\left(\mathbf{U}^{n}\right): \sup _{0 \leq r<1} M_{p}(f, r)<\infty\right\}$, where

$$
\begin{array}{r}
M_{p}^{p}(f, r)=\int_{T^{n}}|f(r \xi)|^{p} d m_{n}(\xi), \\
M_{\infty}(f, r)=\max _{\xi \in T^{n}}|f(r \xi)|, \\
r \in I, \quad f \in H\left(\mathbf{U}^{n}\right) .
\end{array}
$$

As usual, we denote by $\vec{\alpha}$ the vector $\left(\alpha_{1}, \ldots, \alpha_{n}\right)$.

For $\alpha_{j}>-1, j=1, \ldots, n, 0<p<\infty$, recall that the weighted Bergman space $A_{\vec{\alpha}}^{p}\left(\mathbf{U}^{n}\right)$ consists of all holomorphic functions on the polydisk such that

$$
\|f\|_{A_{\vec{\alpha}}^{p}}^{p}=\int_{\mathbf{U}^{n}}|f(z)|^{p} \prod_{i=1}^{n}\left(1-\left|z_{i}\right|^{2}\right)^{\alpha_{i}} d m_{2 n}(z)<\infty .
$$

When $\alpha_{1}=\cdots=\alpha_{n}=\alpha$, then we use notation $A_{\alpha}^{p}\left(\mathbf{U}^{n}\right)$.

If $u$ is $n$-harmonic (harmonic by each variable), then as usual

$$
u\left(r_{1} e^{i \varphi_{1}}, \ldots, r_{n} e^{i \varphi_{n}}\right)=\sum_{k_{1}, \ldots, k_{n}=-\infty}^{\infty} C_{k_{1}, \ldots, k_{n}} \prod_{j=1}^{n} r_{j}^{\left|k_{j}\right|} e^{i k_{j} \varphi_{j}} .
$$

Let further

$$
\begin{aligned}
h^{p}(\vec{\alpha})= & \{u \text { is } n \text {-harmonic: } \\
& \int_{\mathrm{U}^{n}} \prod_{k=1}^{n}\left(1-\left|z_{k}\right|\right)^{\alpha_{k}}\left|u\left(z_{1}, \ldots, z_{n}\right)\right|^{p} d m_{2 n}(z)<\infty, \\
& \left.0<p<\infty, \alpha_{k}>-1, k=1, \ldots, n\right\} .
\end{aligned}
$$

Also, we denote by $\mathbf{B}_{n}$ the unit ball in $\mathbb{C}^{n}, \mathbf{B}_{n}=\{w \epsilon$ $\left.\mathbb{C}^{n}:|w|<1\right\}$. For $1 \leq p<+\infty$ and $\alpha>-1$, denote by $\mathrm{H}_{\alpha}^{\mathrm{p}}\left(\mathrm{B}_{\mathrm{n}}\right)$ (or $A_{\alpha}^{p}\left(B_{n}\right)$ ) the space of all functions $f$ holomorphic in $B_{n}$, satisfying the condition

$$
\int_{\mathbf{B}_{n}}|f(w)|^{p}\left(1-|w|^{2}\right)^{\alpha} d v(w)<+\infty
$$

where $d v$ is the Lebesgue measure in $\mathbb{C}^{n} \equiv \mathbb{R}^{2 n}$ (see [6]).

Further, for a complex number $\beta$ with $\operatorname{Re} \beta>-1$, put

$$
c_{n}(\beta)=\frac{\Gamma(n+1+\beta)}{\pi^{n} \cdot \Gamma(1+\beta)} .
$$

Let $A_{\alpha}^{\infty}\left(\mathbf{B}_{n}\right)=\left\{f \in H\left(\mathbf{B}_{n}\right): \sup _{z \in \mathbf{B}_{n}}|f(z)|(1-|z|)^{\alpha}<\right.$ $\infty\}, \alpha>0$.

Remark 1. Analytic $A_{\alpha}^{\infty}$ spaces are well known in literature as so-called growth spaces (see, e.g., [5]). It can be shown easily that these spaces are Banach spaces. These spaces are playing a vital role in this paper and are embedded in Bergman spaces $A_{\beta}^{p}$, for large enough $\beta$. Hence the representation (10) with $\beta$ large enough index depending on $\alpha$ for all functions from such classes is valid. The mentioned embedding is well known and almost obvious and we leave the proof of it to interested readers. This fact nevertheless is crucial for the proof of Theorems 18 and 20 below and actually serves as base of both proofs, along with well-known Forelli-Rudin estimates for unit ball (see [5]).

We have the following theorem.

Theorem A (see [6]). Assume that $1 \leq p<+\infty, \alpha>-1$ and that the complex number $\beta$ satisfies the condition

$$
\begin{gathered}
\operatorname{Re} \beta \geq \alpha, \quad p=1, \\
\operatorname{Re} \beta>\frac{\alpha+1}{p}-1, \quad 1<p<\infty .
\end{gathered}
$$

Then each function $f \in A_{\alpha}^{p}\left(\mathbf{B}_{n}\right)$ admits the following integral representations:

$$
\begin{gathered}
f(z)=c_{n}(\beta) \cdot \int_{\mathbf{B}_{n}} \frac{f(w)\left(1-|w|^{2}\right)^{\beta}}{(1-\langle z, w\rangle)^{n+1+\beta}} d \nu(w), \quad z \in \mathbf{B}_{n}, \\
\overline{f(0)}=c_{n}(\beta) \cdot \int_{\mathbf{B}_{n}} \frac{\overline{f(w)}\left(1-|w|^{2}\right)^{\beta}}{(1-\langle z, w\rangle)^{n+1+\beta}} d \nu(w), \quad z \in \mathbf{B}_{n},
\end{gathered}
$$


For $n>1$, the theorem was proved in [7] (when $\alpha=0)$ and in $[8,9]$ (when $\alpha>-1)$.

In monographs [5] one can find numerous applications of the formulas (10) and (11) in the complex analysis. In [6] the author generalized Theorem A to the space with anisotropic weight function such as assuming that $1 \leq p<+\infty, \alpha=$ $\left(\alpha_{1}, \ldots, \alpha_{n}\right) \in \mathbb{R}^{n}$ satisfies the conditions

$$
\begin{aligned}
& \alpha_{n}>-1, \\
& \alpha_{n}+\alpha_{n-1}>-2, \\
& \alpha_{n}+\alpha_{n-1}+\alpha_{n-2}>-3, \\
& \vdots \\
& \alpha_{n}+\alpha_{n-1}+\alpha_{n-2}+\cdots+\alpha_{2}>-(n-1), \\
& \alpha_{n}+\alpha_{n-1}+\alpha_{n-2}+\cdots+\alpha_{2}+\alpha_{1}>-n,
\end{aligned}
$$

and then introduced the spaces $A_{\alpha}^{p}\left(\mathbf{B}_{n}\right)$ of functions $f$ holomorphic in $\mathbf{B}_{n}$ satisfying the condition

$$
\begin{gathered}
\int_{\mathbf{B}_{n}}|f(w)|^{p} \prod_{i=1}^{n}\left(1-\left|w_{1}\right|^{2}-\left|w_{2}\right|^{2}-\cdots-\left|w_{i}\right|^{2}\right)^{\alpha_{i}} d \nu(w) \\
<+\infty .
\end{gathered}
$$

For these anisotropic spaces, similarities of the integral representations (10) and (11) are obtained, but in the mentioned generalization special kernels $S_{\beta_{1} \beta_{2} \cdots \beta_{n}}(z, w) \equiv$ $S_{\beta}(z, w)$ (where $\beta_{i}, 1 \leq i \leq n$, are associated with $\alpha_{i}, 1 \leq$ $i \leq n$, and $p$ in a special way) appear instead of $c_{n}(\beta) \cdot(1-$ $\langle z, w\rangle)^{-(n+1+\beta)}$ (see [6], Theorem 4.7).

Suppose now that $\alpha=\left(\alpha_{1}, \alpha_{2}, \ldots, \alpha_{n}\right) \in \mathbb{R}^{n}$. We put

$$
m_{\alpha}=\sum_{\alpha_{i}<0}-\alpha_{i}, \quad l_{\alpha}=\sum_{\alpha_{i}<0} \alpha_{i} .
$$

We will write $\alpha \prec(*)$ only if the following conditions are satisfied:

$$
\alpha_{n}+\alpha_{n-1}+\cdots+\alpha_{i}>-(n+1-i) \quad(1 \leq i \leq n) .
$$

Similarly, if $\beta=\left(\beta_{1}, \beta_{2}, \ldots, \beta_{n}\right) \in \mathbb{C}^{n}$, then we will write $\beta \prec$ $(*)$ if only $\operatorname{Re} \beta \equiv\left(\operatorname{Re} \beta_{1}, \operatorname{Re} \beta_{2}, \ldots, \operatorname{Re} \beta_{n}\right)<(*)$.

We have the following lemmas.

Lemma 2 (see [6]). If $\alpha \prec(*)$, then

$$
\begin{gathered}
\int_{\mathbf{B}_{n}} \prod_{i=1}^{n}\left(1-\left|w_{1}\right|^{2}-\left|w_{2}\right|^{2}-\cdots-\left|w_{i}\right|^{2}\right)^{\alpha_{i}} d \nu(w) \\
=\frac{\pi^{n}}{\prod_{i=1}^{n}\left(\alpha_{n}+\alpha_{n-1}+\cdots+\alpha_{i}+n+1-i\right)}<+\infty .
\end{gathered}
$$

We need also heavily the well-known Forelli-Rudin type estimates for unit ball (see [5]).
Proposition 3. Suppose $c>0$ is real and $t>-1$. Then the integral

$$
J_{c, t}(z)=\int_{\mathbf{B}_{n}} \frac{\left(1-|w|^{2}\right)^{t} d v(w)}{|1-\langle z, w\rangle|^{n+1+t+c}}, \quad z \in \mathbf{B}_{n}
$$

has the following asymptotic property:

$$
J_{c, t} \sim\left(1-|z|^{2}\right)^{-c} \quad \text { as }|z| \longrightarrow 1-.
$$

Definition 4. Assume that $0<p<+\infty, \alpha=\left(\alpha_{1}, \ldots, \alpha_{n}\right) \prec$ $(*)$. Denote by $L_{\alpha}^{p}\left(\mathbf{B}_{n}\right)$ the space of all complex-valued functions $f$ in $\mathbf{B}_{n}$ with

$$
\begin{aligned}
\widetilde{M}_{\alpha}^{p}(f) \equiv & \int_{\mathbf{B}_{n}}|f(w)|^{p} \\
& \quad \times \prod_{i=1}^{n}\left(1-\left|w_{1}\right|^{2}-\left|w_{2}\right|^{2}-\cdots-\left|w_{i}\right|^{2}\right)^{\alpha_{i}} d \nu(w) \\
<\infty &
\end{aligned}
$$

We obviously have

$$
\begin{aligned}
\widetilde{M}_{\alpha}^{p}(f+g) & \leq 2^{p}\left(\widetilde{M}_{\alpha}^{p}(f)+\widetilde{M}_{\alpha}^{p}(g)\right), \\
\widetilde{M}_{\alpha}^{p}(c f) & =c^{p} \widetilde{M}_{\alpha}^{p}(f) .
\end{aligned}
$$

Lemma 5 (see [6]). Assume that $0<p<+\infty, \alpha<(*)$, and $f \in H\left(\mathbf{B}_{n}\right)$. Then

$$
|f(z)|^{p} \leq \frac{2^{2 n+l_{\alpha}}}{c_{n} \cdot(1-|z|)^{2 n+l_{\alpha}}} \cdot \widetilde{M}_{\alpha}^{p}(f) \quad \forall z \in \mathbf{B}_{n}
$$

where $c_{n}=\nu\left(\mathbf{B}_{n}\right)$ is the volume of the unit ball of $\mathbb{C}^{n}$.

Note that this lemma leads to an embedding theorem immediately which allows us to pose a distance problem as unit disk case.

Corollary 6 (see [6]). $H_{\alpha}^{p}\left(\mathbf{B}_{n}\right)$ is a closed subspace in $L_{\alpha}^{p}\left(\mathbf{B}_{n}\right)$.

We will need some facts from theory of Bergman-Orlicz spaces (see $[10,11])$.

Let now $\Psi:[0, \infty) \rightarrow[0, \infty)$ be a continuous nondecreasing function which vanishes and is continuous at 0 . Given a probabilistic space $(\Omega, \mathbb{P})$, we define the Orlicz class $L^{\Omega}(\Omega, \mathbb{P})$ as the set of all (equivalence classes of) measurable functions $f$ on $\Omega$ such that $\int_{\Omega} \Psi(|f| / C) d \mathbb{P}<\infty$ for some $0<C<\infty$. We used to define the Morse-Transue space $M^{\Psi}(\Omega, \mathbb{P})$ by

$$
\begin{aligned}
& M^{\Psi}(\Omega, \mathbb{P})=\{f: \Omega \longrightarrow \mathbb{C} \text { measurable, } \\
&\left.\int_{\Omega} \Psi\left(\frac{|f|}{C}\right) d \mathbb{P}<\infty \text { for any } C>0\right\},
\end{aligned}
$$


and we also introduce the following set:

$$
\begin{aligned}
\mathscr{L}^{\Psi}(\Omega, \mathbb{P}) & \\
& =\left\{f: \Omega \longrightarrow \mathbb{C} \text { measurable, } \int_{\Omega} \Psi(|f|) d \mathbb{P}<\infty\right\} .
\end{aligned}
$$

These three sets are not vector spaces and do not coincide, but we have

$$
M^{\Psi}(\Omega, \mathbb{P}) \subset \mathscr{L}^{\Psi}(\Omega, \mathbb{P}) \subset L^{\Psi}(\Omega, \mathbb{P}) .
$$

We also define the Luxembourg gauge on $L^{\Psi}(\Omega, \mathbb{P})$ by

$$
\|f\|_{\Psi}=\inf \left(\lambda>0, \int_{\Omega} \Psi\left(\frac{|f|}{\lambda}\right) d \mathbb{P} \leq 1\right) .
$$

This functional is homogeneous and is 0 if and only if $f=0$ but it is not subadditive.

We say that the two functions $\Psi_{1}$ and $\Psi_{2}$ as above are equivalent if there exists some constant $C$ such that

$$
C \Psi_{1}(c x) \leq \Psi_{2}(x) \leq C^{-1} \Psi_{1}\left(C^{-1} x\right),
$$

for any $x$ large enough. Two equivalent functions define the same Orlicz class with equivalent Luxembourg functionals.

In order to define a good topology on $L^{\Psi}(\Omega, \mathbb{P})$ and to get properties convenient for our purpose, we will assume that $\Psi$ satisfies the following definition.

Definition 7. Let $0<p \leq 1$. One says that $\Psi:[0, \infty) \rightarrow$ $[0, \infty)$ is a growth function of order $p$ if it satisfies the two following conditions.

(1) $\Psi$ is of lower type $p$, that is, $\Psi(y x) \leq y^{p} \Psi(x)$ for any $0<y \leq 1$ and at least for $x$ large enough.

(2) $x \mapsto \Psi(x) / x$ is nonincreasing, at least for every $x$ large enough.

We will say that $\Psi$ is a growth function if it is a growth function of order $p$ for some $0<p \leq 1$.

Sometimes we will use lower $p$ index for our growth function below.

In particular, a growth function $\Psi$ is equivalent to the function $x \mapsto \int_{0}^{x}(\Psi(s) / s) d s$ which is concave (see [12]). Now, for such a concave growth function of order $p$,

$$
\begin{gathered}
L^{1}(\Omega, \mathbb{P}) \subset M^{\Psi}(\Omega, \mathbb{P})=\mathscr{L}^{\Psi}(\Omega, \mathbb{P})=L^{\Psi} \subset L^{p}(\Omega, \mathbb{P}), \\
\|f\|_{\Psi} \lesssim \min \left\{\int_{\Omega} \Psi(|f|) d \mathbb{P},\left(\int_{\Omega} \Psi(|f|) d \mathbb{P}\right)^{p}\right\},
\end{gathered}
$$

while

$$
\|f\|_{\Psi}:=\int_{\Omega} \Psi(|f|) d \mathbb{P} \lesssim \max \left\{\|f\|_{\Psi},\|f\|_{\Psi}^{p}\right\} .
$$

Moreover, if we define $d_{\Psi}(f, g)=\|f-g\|_{\Psi}\left(d^{\Psi}(f, g)=\right.$ $\|f-g\|^{\Psi}$ ), then $d_{\Psi}$ and $d^{\Psi}$ (that we simply denote by $\|\cdot\|_{\Psi}$ and $\left.\|\cdot\|^{\Psi}\right)$ are two equivalent metrics on $L^{\Psi}(\Omega, \mathbb{P})$ for which it is complete. Without loss of generality, we then assume that every growth function that we consider further is concave and diffeomorphic.

When we deal with spaces of holomorphic functions, it is very natural to require subharmonicity. Then we will assume that $\Psi$ is such that $\Psi(|f|)$ is subharmonic when $f$ is holomorphic. We will refer to such a function as a subharmonic-preserving function.

Here are some examples of (concave) growth function that we may consider further.

For $\Psi$ a subharmonic-preserving growth function and $\alpha>-1$, the weighted Bergman-Orlicz space $A_{\alpha}^{\Psi}$ of the ball consists of those holomorphic functions on $\mathbf{B}_{n}$ which belongs to the Orlicz space $L^{\Psi}\left(\mathbf{B}_{n}, v_{\alpha}\right)$, where, as usual, for any real number $\alpha, d \nu_{\alpha}(z)=c_{\alpha}\left(1-|z|^{2}\right)^{\alpha} d \nu(z)$ for $|z|<1$. Here, if $\alpha \leq-1, c_{\alpha}=1$ and if $\alpha>-1, c_{\alpha}=\Gamma(n+1+\alpha) / \Gamma(n+1) \Gamma(\alpha+1)$ is the normalizing constant so that $\nu_{\alpha}$ has unit total mass.

Further, we will denote by $\|\cdot\|_{\alpha, \Psi}$ the corresponding Luxembourg (quasi)norm and by $\|\cdot\|_{\alpha}^{\Psi}$ the quantity $\int_{\mathbf{B}_{n}} \Psi(|f|) d \nu_{\alpha} . A_{\alpha}^{\Psi}$ is metric space for the distance $d_{\alpha, \Psi}$ or $d_{\alpha}^{\Psi}$ defined by, respectively, $d_{\alpha, \Psi}(f, g)=\|f-g\|_{\alpha, \Psi}$ and $d_{\alpha}^{\Psi}(f, g)=\|f-g\|_{\alpha}^{\Psi}$. If $\Psi(t)=t^{p}$, then we recover the usual weighted Bergman space $A_{\alpha}^{p}$. We have the following inclusions:

$$
A_{\alpha}^{1} \subset A_{\alpha}^{\Psi} \subset A_{\alpha}^{p}
$$

whenever $\Psi$ is a growth function of order $p$.

Note that the second inclusion which we see before leads to another inclusion, namely, $A_{\alpha}^{\Psi}$, is embedded in $A_{(\alpha+n+1) / p}^{\infty}$. This last inclusion will lead us to distance theorem. We showed a complete analogue of a theorem in the unit disk below.

It is important to mention that a linear operator $T$ from $A_{\alpha}^{\Psi_{1}}$ to $X$ with $X=L^{\Psi_{2}}\left(\mathbf{B}_{n}, \nu_{\alpha}\right)$ or $X=A_{\alpha}^{\Psi_{2}}$, where $\Psi_{1}$ and $\Psi_{2}$ are two growth functions, is continuous if and only if it maps a bounded set into a bounded set, or equivalently if and only if there exists a constant $C>0$ such that

$$
\max \left(\|T(f)\|_{\alpha, \Psi_{2}},\|T(f)\|_{\alpha}^{\Psi_{2}}\right) \leq C
$$

for any $f \in A_{\alpha}^{\Psi_{1}}$ such that $\min \left(\|f\|_{\alpha, \Psi_{1}},\|f\|_{\alpha}^{\Psi_{1}}\right) \leq 1$. If $X=\mathbb{C}$, then $T$ is bounded if and only if $|T(f)| \leq C$ for $f$ as previously.

The next proposition says that the point evaluation functionals are continuous on $A_{\alpha}^{\Psi}$.

Proposition 8 (see [11]). Let $\alpha>-1$ and let $\Psi$ be a subharmonic-preserving growth function. For any $\alpha \in \mathbf{B}_{n}$ and any $f \in A_{\alpha}^{\Psi}$, one has

$$
|f(a)| \leq \Psi^{-1}\left(\left(\frac{2}{1-|a|}\right)^{(n+\alpha+1)}\right)\|f\|_{\alpha, \Psi} .
$$

The proof is the same as that of [10, Proposition 1.9] and so is omitted (still use the hypothesis that $\Psi(|f|)$ is subharmonic). We easily deduce from this and the completeness of $L^{\Psi}$ the following result. 
Corollary 9 (see [11]). $A_{\alpha}^{\Psi}$, endowed with $d_{\alpha, \Psi}$ or $d_{\alpha}^{\Psi}$, is a complete metric space.

Some facts from theory of $n$-harmonic function spaces will be needed by us. We will use the same notation for $M_{p}(f, r)$ for harmonic and analytic functions.

Definition 10. The quasinormed space $L(p, q, \alpha)(0<p, q \leq$ $\infty, \alpha=\left(\alpha_{1}, \ldots, \alpha_{n}\right), \alpha_{j}>-1, j=1, \ldots, n$ is the set of those functions $f(z)$ measurable in the polydisc $\mathbf{U}^{n}$, for which the quasinorm

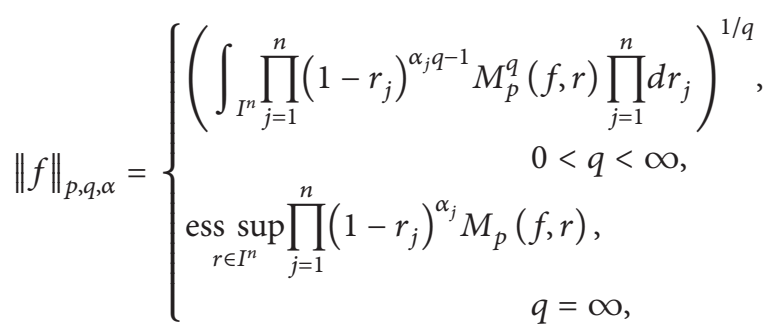

is finite. For the subspace of $L(p, q, \alpha)$ consisting of $n$ harmonic functions let $h(p, q, \alpha)=h\left(\mathbf{U}^{n}\right) \cap L(p, q, \alpha)$ (see $[13])$.

For $p=q<\infty$ the spaces $h(p, q, \alpha)$ coincide with the well-known weighted Bergman spaces, while for $q=\infty$ they are known as growth spaces.

Let now $1 \leq p, q \leq \infty, \alpha_{j}>0$. The following inclusion will be used by us (see [13]):

$$
h(p, q, \alpha) \subset h(\infty, \infty, \alpha) .
$$

This inclusion as in unit disk case (see [2]) allows us to pose a distance problem in spaces of $n$-harmonic functions we consider in this paper (we also did the same in spaces of analytic functions in the unit polydisk (see [1])).

Now we define Poisson kernel $P_{\alpha}$ in the unit disk

$$
P_{\alpha}(z)=\Gamma(\alpha+1)\left[\operatorname{Re} \frac{2}{(1-z)^{\alpha+1}}-1\right], \quad z \in \mathbf{U}, \alpha \geq 0 .
$$

Let $P_{\alpha}(z, \xi)=P_{\alpha}(z \xi)$. For polydisk we have

$$
P_{\alpha}(z, \xi)=\prod_{j=1}^{n} P_{\alpha_{j}}\left(z_{j}, \xi_{j}\right)
$$

where $\alpha=\left(\alpha_{1}, \ldots, \alpha_{n}\right), \alpha_{j} \geq 0, \xi \in \mathbf{U}^{n}, z \in \mathbf{U}^{n} . P_{\alpha}$ is $n$-harmonic by both variables $z$ and $\xi$ and obviously holds $P_{\alpha}(z, \xi)=P_{\alpha}(\xi, z)=P_{\alpha}(\bar{z}, \bar{\xi})$ (see [13]).

Reproducing integral formula Poisson-Bergman type will be presented in the following theorem.
Theorem B (see [13]). Let $\alpha_{j}>0, u \in h(p, q, \alpha)$. If $0<p$, $q \leq \infty, \beta_{j}>\max \left\{\alpha_{j}+1 / p-1, \alpha_{j}\right\}$, or $1 \leq p \leq \infty, 0<q \leq 1$, $\beta_{j} \geq \alpha_{j}(1 \leq j \leq n)$

$$
\begin{aligned}
& u(z)= \frac{1}{\prod_{j=1}^{n} \Gamma\left(\beta_{j}\right)} \\
& \times \int_{\mathbf{U}^{n}} \prod_{j=1}^{n}\left(1-|\zeta|^{2}\right)^{\beta_{j}-1} P_{\beta}(z, \zeta) u(\zeta) d m_{2 n}(\zeta), \\
& z \in \mathbf{U}^{n} .
\end{aligned}
$$

Let $S_{\beta, \lambda}$ be a linear operator Bergman type

$$
\begin{aligned}
& S_{\beta, \lambda}(f)(z) \\
&= \frac{\left(1-|z|^{2}\right)^{\lambda}}{\Gamma(\beta+\lambda)} \\
& \quad \times \int_{\mathbf{U}^{n}}\left(1-|\xi|^{2}\right)^{\beta-1}\left|P_{\beta+\lambda}(z, \xi)\right| f(\xi) d m_{2 n}(\xi) .
\end{aligned}
$$

Theorem C (see [13]). Let $1 \leq p, q \leq \infty, \alpha_{j}, \beta_{j}, \lambda_{j} \in$ $\mathbb{R}, \beta_{j}>\alpha_{j}>-\lambda_{j}, 1 \leq j \leq n$. Then the operator $S_{\beta, \lambda}$ continuously maps the space $L(p, q, \alpha)$ into itself. Moreover, then operator $S_{\beta, \lambda}$ is bounded in $L(p, q, \alpha)$ if and only if $\beta_{j}>$ $\alpha_{j}>-\lambda_{j}, 1 \leq j \leq n$.

We will also need basic facts from theory of Bergman spaces in minimal ball (see $[14,15])$. For the convenience of readers we will give some details about them.

We consider the domain $\mathbb{B}_{*}$ in $\mathbb{C}^{n}, n \geq 2$, defined by

$$
\mathbb{B}_{*}=\left\{z \in \mathbb{C}^{n}:|z|^{2}+|z \cdot z|<1\right\},
$$

where

$$
z \cdot w=\sum_{j=1}^{n} z_{j} w_{j},
$$

for $z$ and $w$ in $\mathbb{C}^{n}$. This is the unit ball of $\mathbb{C}^{n}$ with respect to the norm

$$
N_{*}(z):=\sqrt{|z|^{2}+|z \cdot z|}, \quad z \in \mathbb{C}^{n} .
$$

The norm $N:=N_{*} / \sqrt{2}$ was introduced by Hahn and Pflug in [16], where it was shown to be the smallest norm in $\mathbb{C}^{n}$ that extends the Euclidean norm in $\mathbb{R}^{n}$ under certain restrictions.

The domain $\mathbb{B}_{*}$ has since been studied by Hahn and Pflug [16], Mengotti and Youssfi [15], and Oeljeklaus and Youssfi [17]. In particular, it is well known that the automorphism group of $\mathbb{B}_{*}$ is compact and its identity component is

$$
\operatorname{Aut}_{\mathscr{\mathcal { O }}}^{0}\left(\mathbb{B}_{*}\right)=S^{1} \cdot S O(n, \mathbb{R}),
$$

where the $S^{1}$-action is diagonal and the $S O(n, \mathbb{R})$-action is by matrix multiplication (see [17], e.g.). It is also well known that $\mathbb{B}_{*}$ is a nonhomogeneous domain. Its singular boundary consists of all points $z$ with $z \bullet z=0$, and the regular part of the boundary of $\mathbb{B}_{*}$ consists of strictly pseudoconvex points. 
For each $s>-1$ we let $v_{s}$ denote the measure on $\mathbb{B}_{*}$, defined by

$$
d v_{s}(z):=\left(1-N_{*}^{2}(z)\right)^{s} d v(z)
$$

where $v$ denotes the normalized Lebesgue measure on $\mathbb{B}_{*}$. For all $0<p<\infty$ we define

$$
\mathscr{A}_{s}^{p}\left(\mathbb{B}_{*}\right):=\mathscr{H}\left(\mathbb{B}_{*}\right) \cap L^{p}\left(\mathbb{B}_{*},|z \cdot z|^{(p-2) / 2} d v_{s}\right),
$$

where $\mathscr{H}\left(\mathbb{B}_{*}\right)$ is the space of all holomorphic functions on $\mathbb{B}_{*}$. Naturally, the spaces $\mathscr{A}_{s}^{P}\left(\mathbb{B}_{*}\right)$ are called weighted Bergman spaces of $\mathbb{B}_{*}$.

When $p=2$, there exists an orthogonal projection from $L^{2}\left(\mathbb{B}_{*}, d v_{s}\right)$ onto $\mathscr{A}_{s}^{2}\left(\mathbb{B}_{*}\right)$. This will be called the weighted Bergman projection and is denoted by $\mathbf{P}_{s, \mathbb{B}_{*}}$.

It is well known that $\mathbf{P}_{s, \mathbb{B}_{*}}$ is an integral operator on $L^{2}\left(\mathbb{B}_{*}, d v_{s}\right)$, namely,

$$
\mathbf{P}_{s, \mathbb{B}_{*}} f(z)=\int_{\mathbb{B}_{*}} \mathbf{K}_{s, \mathbb{B}_{*}}(z, w) f(w) d v_{s}(w),
$$

where

$$
\mathbf{K}_{s, \mathbb{B}_{*}}(z, w)=\frac{\mathbf{A}(X, Y)}{\left(n^{2}+n-s\right) v_{s}\left(\mathbb{B}_{*}\right)\left(X^{2}-Y\right)^{n+1+s}}
$$

is the weighted Bergman kernel. Here

$$
X=1-z \cdot \bar{w}, \quad Y=(z \cdot z) \overline{w \cdot w},
$$

and $\mathbf{A}(X, Y)$ is the sum

$$
\begin{aligned}
& \sum_{j=0}^{\infty} c_{n, s, j} X^{n+s-1-2 j} Y^{j} \\
& \quad \times\left[2(n+s) X-\frac{(n-2 j+s)(n+1+2 s)}{n+s+1}\left(X^{2}-Y\right)\right] .
\end{aligned}
$$

As usual,

$$
\begin{aligned}
c_{n, s, j} & =\left(\begin{array}{c}
n+s+1 \\
2 j+1
\end{array}\right) \\
& =\frac{(n+s+1)(n+s) \cdots(n+s-2 j+1)}{(2 j+1) !}
\end{aligned}
$$

is the binomial coefficient and $v_{s}\left(\mathbb{B}_{*}\right)$ is the weighted Lebesgue volume of $\mathbb{B}_{*}$. See $[15,17]$ for these formulas and more information about the weighted Bergman kernels.

Because of the infinite sum $A(X, Y)$ the formula for $\mathbf{K}_{s, \mathbb{B}_{*}}$ is not really a closed form. As such, it is inconvenient for us to do estimates for $\mathbf{P}_{s, \mathbb{B}_{*}}$ directly. Thus we employ a technique that was used in [15]. More specifically, we relate the domain $\mathbb{B}_{*}$ to the hypersurface $\mathbb{M}$ of the Euclidean unit ball in $\mathbb{C}^{n+1}$ defined by

$$
\mathbb{M}=\left\{z \in \mathbb{C}^{n+1} \backslash\{0\}: z \cdot z=0,|z|<1\right\} .
$$

If $\operatorname{Pr}: \mathbb{C}^{n+1} \rightarrow \mathbb{C}^{n}$ is defined by

$$
\operatorname{Pr}\left(z_{1}, \ldots, z_{n}, z_{n+1}\right)=\left(z_{1}, \ldots, z_{n}\right) \text {, }
$$

and $\mathbf{F}=\operatorname{Pr}_{\mid \mathbb{M}}$, then $\mathbf{F}: \mathbb{M} \rightarrow \mathbb{B}_{*}-\{0\}$. Let

$$
\mathbb{M}=\left\{z \in \mathbb{C}^{n+1} \backslash\{0\}: z \cdot z=0\right\} .
$$

It was proved in [17] that there is an $S O(n+1, \mathbb{C})$-invariant

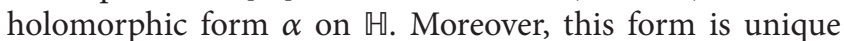
up to a multiplicative constant. In fact, after appropriate normalization, the restriction to $\mathbb{H} \cap(\mathbb{C} \backslash\{0\})^{n+1}$ of this form is given by

$$
\alpha(z)=\sum_{j=1}^{n+1} \frac{(-1)^{j-1}}{z_{j}} d z_{1} \wedge \cdots \wedge \widehat{d z}_{j} \wedge \cdots \wedge d z_{n+1} .
$$

Our norm estimates for the weighted Bergman projection $\mathbf{P}_{s, \mathbb{B}_{*}}$ on $L^{p}$ spaces will be based on the corresponding estimates on $\mathbb{M}$. Therefore, we will consider the spaces $L_{s}^{p}(\mathbb{M})$ consisting of measurable complex-valued functions $f$ on $\mathbb{M}$ such that

$$
\|f\|_{L_{s}^{p}(\mathbb{M})}^{p}=\int_{\mathbb{M}}|f(z)|^{p}\left(1-|z|^{2}\right)^{s} \frac{\alpha(z) \wedge \bar{\alpha}(z)}{\bar{C}}<\infty,
$$

where

$$
\bar{C}:=(-1)^{n(n+1) / 2}(2 i)^{n} .
$$

We use $A_{s}^{P}(\mathbb{M})$ to denote the subspace of all holomorphic functions in $L_{s}^{p}(\mathbb{M})$. The weighted Bergman projection $\mathbf{P}_{s, \mathbb{M}}$ is then the orthogonal projection from $L_{s}^{2}(\mathbb{M})$ onto $A_{s}^{2}(\mathbb{M})$. Again, it is well known that $\mathbf{P}_{s, \mathbb{M}}$ is an integral operator on $L_{s}^{2}(\mathbb{M})$ given by the formula

$$
\mathbf{P}_{s, \mathbb{M}} f(z)=\int_{\mathbb{M}} \mathbf{K}_{s, \mathbb{M}}(z, w) f(w)\left(1-|w|^{2}\right)^{s} \frac{\alpha(z) \wedge \bar{\alpha}(z)}{\bar{C}},
$$

where $\mathbf{K}_{s, \mathbb{M}}$ is the corresponding Bergman kernel.

The starting point for our analysis is the following closed form of $\mathbf{K}_{s, \mathbb{M}}$, which was obtained as Theorem 3.2 in [15].

Lemma 11 (see [14]). The weighted Bergman kernel $\mathbf{K}_{s, \mathbb{M}}$ of $A_{s}^{2}(\mathbb{M})$ is given by

$$
\mathbf{K}_{s, \mathbb{M}}(z, w)=\frac{C(n-1+(n+1+2 s) z \cdot \bar{w})}{(1-z \cdot \bar{w})^{n+s+1}},
$$

where $C$ is a certain constant that depends on $n$ and $s$.

Let $f: \mathbb{B}_{*} \rightarrow \mathbb{C}$ be a measurable function. We define a function $\mathbf{T} f$ on $\mathbb{M}$ by

$$
\begin{aligned}
(\mathbf{T} f)(z): & =\frac{z_{n+1}}{\left(2(n+1)^{2}\right)^{1 / p}}(f \circ \mathbf{F})(z) \\
& =\frac{z_{n+1} f\left(z_{1}, \ldots, z_{n}\right)}{\left(2(n+1)^{2}\right)^{1 / p}} .
\end{aligned}
$$


The operator $\mathbf{T}$ will also play a key role in our analysis. In particular, we need the following result which was obtained as Lemma 4.1 in [10].

Lemma 12 (see [14]). For each $p \geqslant 1$ and $s>-1$ the linear operator $\mathbf{T}$ is an isometry from $L^{p}\left(\mathbb{B}_{*},|z \cdot z|^{(p-2) / 2} d v_{s}\right)$ to $L_{s}^{p}(\mathbb{M})$. Moreover, we have $\mathbf{P}_{s, \mathbb{M}} \mathbf{T}=\mathbf{T P}_{s, \mathbb{B}_{*}}$ on $L^{p}\left(\mathbb{B}_{*},|z \cdot z|^{(p-2) / 2} d v_{s}\right)$.

Theorem B of [15] states that the operator with positive kernel $\mathbf{P}_{s, \mathbb{B}_{*}}$ maps $L^{p}\left(\mathbb{B}_{*},|z \cdot z|^{(p-2) / 2} d v_{s}\right)$ boundedly onto $\mathscr{A}_{s}^{p}\left(\mathbb{B}_{*}\right)$ for all $p>1$. We need to derive an improved version of the classical Forelli-Rudin integral estimates in the case of the minimal ball.

Lemma 13 (see [14]). Suppose $T>0, A>-1$, and $d$ is a nonnegative integer. Then there exists a constant $C>0$ (depending on $d, T$, and $A$, but not on $t$ and s) such that

$$
\begin{gathered}
\int_{\mathbb{M}} \frac{|z \cdot \bar{w}|^{2 d}}{|1-z \cdot \bar{w}|^{n+t+s+1}}\left(1-|w|^{2}\right)^{s} \alpha(w) \wedge \bar{\alpha}(w) \\
\leqslant \frac{C \Gamma(s+1) \Gamma(t)}{\left(1-|z|^{2}\right)^{t}}
\end{gathered}
$$

for all $-1<s<A, 0<t<T$, and $z \in \mathbb{M}$.

For any $s>-1$ we consider the measure

$$
\begin{aligned}
& d \lambda_{s}(z) \\
& \quad=\frac{\Gamma(n+s)}{2 \omega(\partial \mathbb{M}) \Gamma(n-1) \Gamma(s+1)}\left(1-|z|^{2}\right)^{s} \alpha(z) \wedge \bar{\alpha}(z),
\end{aligned}
$$

on $\mathbb{M}$, where $w$, $\omega$ is the $(2 n-1)$-form on $\partial \mathbb{M}$ defined by

$$
\omega(z)\left(V_{1}, \ldots, V_{2 n-1}\right)=\alpha(z) \wedge \bar{\alpha}(z)\left(z, V_{1}, \ldots, V_{2 n-1}\right) .
$$

Proposition 14 (see [14]). Suppose $1 \leq p<\infty$ and $-1<s<$ $\infty$. Then

$$
|g(z)|^{p} \leq \frac{1}{\left(1-|z|^{2}\right)^{n+1+s}} \int_{\mathbb{M}}|g(w)|^{p} d \lambda_{s}(w)
$$

for all $g \in \mathscr{A}_{s}^{p}(\mathbb{M})$ and $z \in \mathbb{M}$.

This proposition leads directly to embeddings between $\mathscr{A}_{s}^{\infty}$ and $\mathscr{A}_{s_{1}}^{p}$ for some $s$ and $s_{1}$ as we had above for unit disk case (see [2]) and we showed that this repeating arguments of unit disk case leads to new theorem we formulate below, in next section, for Bergman spaces in minimal ball.

\section{On Distances in $K$-Bergman Spaces, Large Bergman-Orlicz Spaces in $\mathbb{C}^{n}$, and Bergman Spaces in Minimal Ball}

Based fully on preliminaries of previous section, the plan of this section is the following: first we formulate a result in the unit disk and then repeat arguments we provided in proof of that theorem in various situations in higher dimensions. Since all proofs are short, the repetitions of arguments which are needed in higher dimension will be omitted; only sketches will be given.

It is well known that $A_{-s q-1}^{q}(\mathbf{U}) \subset A_{-t}^{\infty}(\mathbf{U}), t=s-1 / q, t<$ $0, s<0$ (see [18]).

It is easy to note, based on previous section results, that the complete analogues of the embedding we just provided are valid also in Bergman spaces in minimal ball for $n$ harmonic function spaces in polydisk and in $K$-Bergman spaces, and this is based completely on preliminaries we have in previous section. We leave this easy task to readers. This allows us to pose a distance problem in each space we consider in this paper.

In the following theorem (see [1]) we calculate distances from a weighted Bloch class to Bergman spaces for $q \leq 1$. It is easy to note that each argument below is also valid not only in unit disk but based on preliminaries in the previous section in Bergman spaces in minimal ball and polydisk and $K$ Bergman spaces and Bergman-Orlicz spaces. So this theorem is very typical for us though it is known.

Theorem 15. Let $0<q \leq 1, s<0, t \leq s-1 / q, \beta>(1-$ $s q) / q-2$, and $\beta>-1-t$. Let $f \in A_{-t}^{\infty}$. Then the following are equivalent:

(a) $l_{1}=\operatorname{dist}_{A_{-t}^{\infty}}\left(f, A_{-s q-1}^{q}\right)$

(b) $l_{2}=\inf \left\{\varepsilon>0: \int_{\mathbf{U}}\left(\int_{\Omega_{\varepsilon, t}(f)}\left((1-|w|)^{\beta+t} / \mid 1-\right.\right.\right.$ $\left.\left.\left.\left.\langle\bar{z}, w\rangle\right|^{2+\beta}\right) d m_{2}(w)\right)^{q}(1-|z|)^{-s q-1} d m_{2}(z)<\infty\right\}$.

Proof. First we show that $l_{1} \leq C l_{2}$. For $\beta>-1-t$, we have

$$
\begin{aligned}
f(z)=C(\beta)\left(\int_{\mathbf{U} \backslash \Omega_{\varepsilon,-t}} \frac{f(w)(1-|w|)^{\beta}}{(1-\langle\bar{w}, z\rangle)^{\beta+2}} d m_{2}(w)\right. \\
\left.\quad+\int_{\Omega_{\varepsilon,-t}} \frac{f(w)(1-|w|)^{\beta}}{(1-\langle\bar{w}, z\rangle)^{\beta+2}} d m_{2}(w)\right) \\
=f_{1}(z)+f_{2}(z),
\end{aligned}
$$

where $C(\beta)$ is a well-known Bergman representation constant (see $[5,18])$.

For $t<0$,

$$
\begin{aligned}
\left|f_{1}(z)\right| & \leq C \int_{\mathbf{U} \backslash \Omega_{\varepsilon,-t}} \frac{|f(w)|(1-|w|)^{\beta}}{|1-\langle\bar{w}, z\rangle|^{\beta+2}} d m_{2}(w) \\
& \leq C \varepsilon \int_{\mathbf{U}} \frac{(1-|w|)^{\beta+t}}{|1-\langle\bar{w}, z\rangle|^{\beta+2}} d m_{2}(w) \\
& \leq C \varepsilon \frac{1}{(1-|z|)^{-t}} .
\end{aligned}
$$

So $\sup _{z \in \mathbf{U}}\left|f_{1}(z)\right|(1-|z|)^{-t}<C \varepsilon$. 
For $s<0, t<0$, we have

$$
\begin{aligned}
& \int_{\mathbf{U}}\left|f_{2}(z)\right|^{q}(1-|z|)^{-s q-1} d m_{2}(z) \\
& \quad \leq C \int_{\mathbf{U}}\left(\int_{\Omega_{\varepsilon, t}} \frac{(1-|w|)^{\beta+t}}{|1-\langle\bar{w}, z\rangle|^{\beta+2}} d m_{2}(w)\right)^{q}
\end{aligned}
$$

So we finally have

$$
\operatorname{dist}_{A_{-t}^{\infty}}\left(f, A_{-s q-1}^{q}\right) \leq C\left\|f-f_{2}\right\|_{A_{-t}^{\infty}}=C\left\|f_{1}\right\|_{A_{-t}^{\infty}} \leq C \varepsilon .
$$

It remains to prove that $l_{2} \leq l_{1}$. Let us assume that $l_{1}<l_{2}$. Then we can find two numbers $\varepsilon, \varepsilon_{1}$ such that $\varepsilon>\varepsilon_{1}>0$, and a function $f_{\varepsilon_{1}} \in A_{-s q-1}^{q},\left\|f-f_{\varepsilon_{1}}\right\|_{A_{-t}^{\infty}} \leq$ $\varepsilon_{1}$, and $\int_{\mathrm{U}}\left(\int_{\Omega_{\varepsilon,-t}}\left((1-|w|)^{\beta+t} /|1-\langle\bar{z}, w\rangle|^{\beta+2}\right) d m_{2}(w)\right)^{q}(1-$ $|z|)^{-s q-1} d m_{2}(z)=\infty$. Hence as above we easily get from $\left\|f-f_{\varepsilon_{1}}\right\|_{A_{-t}^{\infty}} \leq \varepsilon_{1}$ that $\left(\varepsilon-\varepsilon_{1}\right) \chi_{\Omega_{\varepsilon,-t}(f)}(z)(1-|z|)^{t} \leq C\left|f_{\varepsilon_{1}}(z)\right|$, and hence

$$
\begin{aligned}
M= & \int_{\mathbf{U}}\left(\int_{\mathbf{U}} \frac{\chi_{\Omega_{\varepsilon,-t}(f)}(z)(1-|w|)^{\beta+t}}{|1-\langle\bar{w}, z\rangle|^{\beta+2}} d m_{2}(w)\right)^{q} \\
& \times(1-|z|)^{-s q-1} d m_{2}(z) \\
\leq C & \int_{\mathbf{U}}\left(\int_{\mathbf{U}} \frac{\left|f_{\varepsilon_{1}}(w)\right|(1-|w|)^{\beta}}{|1-\langle\bar{w}, z\rangle|^{\beta+2}} d m_{2}(w)\right)^{q} \\
& \times(1-|z|)^{-s q-1} d m_{2}(z) .
\end{aligned}
$$

Since for $q \leq 1($ see $[5,18])$

$$
\begin{aligned}
& \left(\int_{\mathrm{U}} \frac{\left|f_{\varepsilon_{1}}(z)\right|(1-|z|)^{\alpha} d m_{2}(z)}{|1-\langle w, z\rangle|^{t}}\right)^{q} \\
& \quad \leq C \int_{\mathrm{U}} \frac{\left|f_{\varepsilon_{1}}(z)\right|^{q}(1-|z|)^{\alpha q+q-2} d m_{2}(z)}{|1-\langle w, z\rangle|^{t q}},
\end{aligned}
$$

where $\alpha>(1-q) / q, t>0, f_{\varepsilon_{1}} \in H(\mathbf{U}), w \in \mathbf{U}$, and

$$
\int_{\mathbf{U}} \frac{(1-|z|)^{-s q-1}}{|1-\langle\bar{w}, z\rangle|^{q(\beta+2)}} d m_{2}(z) \leq \frac{C}{(1-|w|)^{q(\beta+2)+s q-1}},
$$

where $s<0, \beta>((1-s q) / q)-2$, and $w \in \mathbf{U}$. We get

$$
M \leq C \int_{\mathrm{U}}\left|f_{\varepsilon_{1}}(z)\right|^{q}(1-|z|)^{-s q-1} d m_{2}(z) .
$$

So, we arrive at a contradiction. The theorem is proved.

The following theorem is a version of Theorem 15 for the case $q>1$.

Theorem 16. Let $q>1, s<0, t \leq s-1 / q, \beta>(-1-s q) / q$, and $\beta>-1-t$. Let $f \in A_{-t}^{\infty}$. Then the following are equivalent:

(a) $l_{1}=\operatorname{dist}_{A_{-t}^{\infty}}\left(f, A_{-s q-1}^{q}\right)$,

$$
\begin{gathered}
\text { (b) } \widehat{l}_{2}=\inf \left\{\varepsilon>0: \int_{\mathbf{U}}\left(\int _ { \Omega _ { \varepsilon , t } ( f ) } \left((1-|w|)^{\beta+t} /\right.\right.\right. \\
\left.\left.\left.|1-\bar{z} w|^{2+\beta}\right) d m_{2}(w)\right)^{q}(1-|z|)^{-s q-1} d m_{2}(z)<\infty\right\} .
\end{gathered}
$$

The proof of Theorem 16 is the same actually as the proof of Theorem 15. The only difference is the boundedness of Bergman type projection operator but with positive Bergman kernel. This fact will be used by us below heavily.

Indeed the close inspection of the proof of Theorem 15 shows that the proof of Theorem 16 is the same as the proof of Theorem 15. but here we will use (70) (see below) instead of (67). For $\varepsilon>0, q>1, \beta>0, \alpha>-1 / q$ (see [18])

$$
\begin{aligned}
& \left(\int_{\mathbf{U}} \frac{|f(z)|(1-|z|)^{\alpha}}{|1-\bar{w} z|^{\beta+2}} d m_{2}(z)\right)^{q} \\
& \leq C \int_{\mathbf{U}} \frac{|f(z)|^{q}(1-|z|)^{\alpha q}}{|1-\bar{w} z|^{\beta q-\varepsilon q+2}} d m_{2}(z)(1-|w|)^{-\varepsilon q}, \quad w \in \mathbf{U},
\end{aligned}
$$

which follows immediately from Hölder's inequality and (68) (see [18]).

We formulate one more theorem in this direction.

Theorem 17. Let $\beta_{j}>\beta_{0}, j=1, \ldots, n$, for large enough $\beta_{0}$, $p>1$, and $\alpha_{j}>0, j=1, \ldots, n$. Then for $f \in h(\infty, \infty, \vec{\alpha})$ we have

$$
\begin{aligned}
\operatorname{dist}_{h(\infty, \infty, \vec{\alpha})}(f, h(p, p, \vec{\alpha})) \\
\approx \inf \{\varepsilon>0: \\
\int_{\mathbf{U}^{n}}\left(\int_{\Omega_{\varepsilon, \vec{\alpha}}^{0}} \prod_{j=1}^{n}\left|P_{\beta_{j}}\left(z_{j}, w_{j}\right)\right||f(w)|\right. \\
\left.\times \prod_{k=1}^{n}\left(1-\left|z_{k}\right|\right)^{\alpha_{k}} d m_{2 n}(z)<\infty\right\},
\end{aligned}
$$

where

$$
\begin{array}{r}
\Omega_{\varepsilon, \tilde{t}}^{0}=\left\{z \in \mathbf{U}^{n}:|f(z)| \prod_{k=1}^{n}\left(1-\left|z_{k}\right|\right)^{\tilde{t}} \geq \varepsilon\right\}, \\
\tilde{t} \geq 0, \quad \varepsilon>0 .
\end{array}
$$

Note also that now even a more general theorem for all mixed norm spaces is valid, namely, for $h(p, q, \alpha)$ spaces based on facts we provided already above concerning projection theorems. We leave this to interested readers.

The scheme of proofs of all high dimensional theorems of this section is the same. The main tools are (see $[1,2])$ the Bergman representation formula for larger space (for each $f$ function from $Y, f \in Y, X \subset Y)$. Then we use the fact that $\||P| f\|_{X} \leq C\|f\|_{X}, f \in X$, where $|P|$ is a Bergman 
projection with positive Bergman kernel. Then we use ForelliRudin type estimate. As example we provide a complete proof of Theorem 17. For a parallel proof of other assertions we refer the reader to facts we provided above taken from mentioned papers and leave this procedure to readers.

Now we return to the proof of Theorem 17. We follow arguments of unit disk case.

Proof of Theorem 17. Let $\sup _{r \in I^{n}}\left(\prod_{j=1}^{n}\left(1-r_{j}\right)^{\alpha_{j}}\right) M_{\infty}(f, r)<$ $\infty$.

Then $\int_{\mathbf{U}^{n}}|f(z)|^{p}\left(\prod_{j=1}^{n}\left(1-\left|z_{j}\right|\right)^{\beta_{j}}\right) d m_{2 n}(z)<\infty$ for all $\beta_{j}>\beta_{0}, j=1, \ldots, n$, where $\beta_{0}$ is large enough. This means that based on lemmas on $n$-harmonic functions from the previous section that for large enough $\widetilde{\beta}_{j}, \widetilde{\beta}_{j}>\widetilde{\beta}_{0}, j=$ $1, \ldots, n$,

$$
\begin{aligned}
f(z)= & \frac{1}{\prod_{j=1}^{n} \Gamma\left(\widetilde{\beta}_{j}\right)} \\
& \times \int_{\mathbf{U}^{n}}\left(\prod_{j=1}^{n}\left(1-\left|\xi_{j}\right|\right)^{\widetilde{\beta}_{j}-1}\right) \\
& \quad \times P_{\overrightarrow{\widehat{\beta}}}(z, \xi) f(\xi) d m_{2 n}(\xi), \quad z \in \mathbf{U}^{n}, \\
& \left|P_{\vec{\alpha}}(z, \xi)\right| \\
& \leq C(\alpha, n) \prod_{j=1}^{n} \frac{1}{\left|1-\overline{\xi_{j}} z_{j}\right|^{\alpha_{j}+1}}, \quad z, \xi \in \mathbf{U}^{n}, \alpha_{j} \geq 0 .
\end{aligned}
$$

Then we have $\widetilde{\beta}_{j}>\widetilde{\beta}_{0}, j=1, \ldots, n: f=f_{1}+f_{2}$ as in unit disk case,

$$
\begin{aligned}
& \left|f_{1}(z)\right| \leq C_{1} \varepsilon \frac{1}{\prod_{j=1}^{n}\left(1-\left|z_{j}\right|\right)^{\alpha}}, \\
& \left|f_{2}(z)\right| \leq C_{2}\|f\|_{h(\infty, \infty, \alpha)} .
\end{aligned}
$$

So finally we have $\operatorname{dist}_{h(\infty, \infty, \alpha)}\left(f_{2}, h(p, \alpha)\right) \leq$ $C\left\|f-f_{2}\right\|_{h(\infty, \infty, \alpha)}=C\left\|f_{1}\right\|_{h(\infty, \infty, \alpha)}$.

To get the reverse we again repeat and follow arguments of unit disk case and use the fact from the previous section

$$
\begin{aligned}
& S_{\widetilde{\beta}_{0}} f(z) \\
& =C\left(\widetilde{\beta}_{0}\right) \int_{\mathbf{U}^{n}} \prod_{j=1}^{n}\left(1-\left|\xi_{j}\right|^{2}\right)^{\widetilde{\beta}_{0}-1}\left|P_{\widetilde{\beta}_{0}}(z, \xi)\right| f(\xi) d m_{2 n}(\xi)
\end{aligned}
$$

maps for large enough $\widetilde{\beta}_{0}$ and for all $\alpha_{j}>0,1 \leq p, q \leq$ $\infty, j=1, \ldots, n, L(p, q, \alpha)$ into $L(p, q, \alpha)$.

The following theorem is based heavily on Remark 1 , which we provided above, and Forelli-Rudin type estimates for unit ball (see [5] and Proposition 3) and some elementary arguments we see in proof of unit disk case.

We formulate complete analogue of Theorem 15 in $K$ Bergman spaces.
Theorem 18. Let $\beta>\beta_{0}$ for large enough $\beta_{0}, \vec{\alpha} \prec(*), 0<$ $p<\infty, l_{\alpha}=\sum_{i>0} \alpha_{i}$. Let $g \in H\left(\mathbf{B}_{n}\right)$. If $\left\|\left|P_{\beta}\right| g\right\|_{A_{\vec{\alpha}}^{p}} \leq$ $C\|g\|_{A_{\vec{\alpha}}^{p}} \Leftrightarrow$

$$
\begin{aligned}
& \int_{\mathbf{B}_{n}}\left(\int_{\mathbf{B}_{n}} \frac{|g(w)|(1-|w|)^{\beta}}{|1-\langle z, w\rangle|^{n+1+\beta}} d v(w)\right)^{p} \\
& \cdot \prod_{i=1}^{n}\left(1-\sum_{j=1}^{i}\left|z_{j}\right|^{2}\right)^{\alpha_{i}} d \nu(z) \\
& \leq C \int_{\mathbf{B}_{n}}|g(w)|^{p} \cdot \prod_{i=1}^{n}\left(1-\sum_{j=1}^{i}\left|w_{j}\right|^{2}\right)^{\alpha_{i}} d \nu(w),
\end{aligned}
$$

then for $f \in A_{\left(2 n+l_{\alpha}\right) / p}^{\infty}\left(\mathbf{B}_{n}\right)$ we have

$$
\begin{aligned}
& \operatorname{dist}_{A_{\left(2 n+l_{\alpha}\right) / p}^{\infty}\left(\mathbf{B}_{n}\right)}\left(f, H_{\alpha_{1}, \ldots, \alpha_{n}}^{p}\left(\mathbf{B}_{n}\right)\right) \\
& \approx \inf \left\{\varepsilon>0: \int_{\mathbf{B}_{n}}\left(\int_{\Omega_{\varepsilon, t}^{1}(f)} \frac{(1-|w|)^{\beta+t} d \nu(w)}{|1-\langle z, w\rangle|^{n+1+\beta}}\right)^{p}\right. \\
& \left.\cdot \prod_{i=1}^{n}\left(1-\sum_{j=1}^{i}\left|w_{j}\right|^{2}\right)^{\alpha_{i}} d \nu(z)<\infty\right\},
\end{aligned}
$$

where

$$
\begin{array}{r}
\Omega_{\varepsilon, \tilde{t}}^{1}(f)=\left\{z \in \mathbf{B}_{n}:|f(z)|(1-|z|)^{\tilde{t}}>\varepsilon\right\}, \\
\varepsilon>0, \quad \tilde{t}>0, \quad \tilde{t}=\frac{2 n+l_{\alpha}}{p} .
\end{array}
$$

Remark 19. Note that the condition in Theorem 18 is true when $\alpha_{j}=0, \alpha_{n} \neq 0$ or $\alpha_{j}=0, j=1, \ldots, n$. This is a classical fact of theory of Bergman spaces in the unit ball (see [5]).

The following theorem is based heavily on Remark 1, which we provided above, and Forelli-Rudin type estimates for unit ball (see [5] and Proposition 3) and some elementary arguments we see in proof of unit disk case.

We formulate complete analogue of Theorem 15 in Bergman-Orlicz spaces.

Theorem 20. Let $\alpha>-1,0<p<\infty$. Then for $f \in$ $A_{(n+1+\alpha) / p}^{\infty}\left(\mathbf{B}_{n}\right)$ we have

$$
\begin{aligned}
& \operatorname{dist}_{A_{(n+1+\alpha) / p}^{\infty}\left(\mathbf{B}_{n}\right)}\left(f, A_{\alpha}^{\Psi_{p}}\left(\mathbf{B}_{n}\right)\right) \\
& =\left\{\varepsilon>0: \int_{\mathbf{B}_{n}} \Psi_{p}\left(\int_{\Omega_{\varepsilon,(n+1+\alpha) / p}^{2}} \frac{(1-|w|)^{\beta-(n+1+\alpha) / p} d \nu(w)}{|1-\langle z, w\rangle|^{n+1+\beta}}\right) d v_{\alpha}\right. \\
& <\infty\},
\end{aligned}
$$


where

$$
\Omega_{\varepsilon, \tilde{t}}^{2}=\left\{z \in \mathbf{B}_{n}:|f(z)|(1-|z|)^{\tilde{t}}>\varepsilon\right\}, \quad \varepsilon>0, \tilde{t}>0,
$$

if $\left\|\left|P_{\beta}\right| g\right\|_{L_{\tilde{\alpha}}^{\Psi_{p}}} \leq C\|g\|_{L_{\tilde{\alpha}}^{\Psi_{p}}}$ for $\left.g \in A_{\tilde{\alpha}}^{\Psi_{p}}\left(\mathbf{B}_{n}\right)\right), \tilde{\alpha}>-1, \beta>\beta_{0}$, for large enough $\beta_{0}$, where $P_{\beta}$ is a weighted Bergman projection in Bergman-Orlicz spaces.

We formulate complete analogue of Theorem 15 in minimal ball.

Theorem 21. Let $\beta>\beta_{0}$ for large enough $\beta_{0}, 1<p<$ $\infty,-1<s<\infty$. Then for any $f \in A_{(n+1+s) / p}^{\infty}(M)$ for which Bergman representation (55) is valid

$$
\begin{aligned}
& \operatorname{dist}_{A_{(n+1+s) / p}^{\infty}(M)}\left(f, A_{s}^{p}(M)\right) \\
& =\inf \left\{\varepsilon>0: \int_{M}\left(\int_{\Omega_{\varepsilon,(n+1+s) / p}^{3}}\left|K_{\beta, M}(z, w)\right| f(w)\right.\right. \\
& \left.\left.\times(1-|w|)^{\beta+t} d \lambda(w)\right)^{p} d \lambda_{s}(w)<\infty\right\},
\end{aligned}
$$

where $t=-(n+1+s) / p$ and

$$
\Omega_{\varepsilon, \tilde{t}}^{3}=\left\{z \in M:|f(z)|(1-|z|)^{\tilde{t}} \geq \varepsilon\right\}, \quad \tilde{t}>0, \varepsilon>0 .
$$

\section{Conflict of Interests}

The authors declare that there is no conflict of interests regarding the publication of this paper.

\section{Acknowledgment}

This paper is supported by MNTR Serbia, Project 174017.

\section{References}

[1] R. Shamoyan and O. R. Mihić, "On new estimates for distances in analytic function spaces in the unit disk, the polydisk and the unit ball," Boletín de la Asociación Matemática Venezolana, vol. 17, no. 2, pp. 89-103, 2010.

[2] R. F. Shamoyan and O. R. Mihić, "On new estimates for distances in analytic function spaces in higher dimension," Siberian Electronic Mathematical Reports, vol. 6, pp. 514-517, 2009.

[3] R. F. Shamoyan, "On an extremal problem in analytic function spaces in tube domains over symmetric cones," to appear in Issues of Analysis.

[4] R. F. Shamoyan, "On an extremal problem in analytic spaces in two Siegel domains in $\mathbb{C}^{n}$," ROMAI Journal, vol. 8, no. 2, pp. 167180, 2012.

[5] F. A. Shamoian and M. Djrbashian, Topics in the Theory of Bergman $A_{\alpha}^{p}$ Spaces, Teubner-Texte zur Mathematik, Teubner, Leipzig, Germany, 1988.
[6] A. Karapetyan, "Weighted anisotropic integral representations of holomorphic functions in the unit ball of $\mathbb{C}^{n}$," Abstract and Applied Analysis, vol. 2010, Article ID 354961, 23 pages, 2010.

[7] F. Forelli and W. Rudin, "Projections on spaces of holomorphic functions in balls," Indiana University Mathematics Journal, vol. 24, pp. 593-602, 1975.

[8] M. Djrbashian, "Survey of some achievements of Armenian mathematicians in the theory of integral representations and factorization of analytic functions," Matematički Vesnik, vol. 39, no. 3, pp. 263-282, 1987.

[9] M. Djrbashian, "A brief survey of the results of research by Armenian mathematicians in the theory of factorization of meromorphic functions and its applications," Journal of Contemporary Mathematical Analysis, vol. 23, no. 6, pp. 8-34, 1988.

[10] S. Charpentier, "Composition operators on weighted BergmanOrlicz spaces on the ball," Complex Analysis and Operator Theory, vol. 7, no. 1, pp. 43-68, 2011.

[11] S. Charpentier and B. Sehba, "Carleson measure theorems for large Hardy-Orlicz and Bergman-Orlicz spaces," Journal of Function Spaces and Applications, vol. 2012, Article ID 792763, 21 pages, 2012.

[12] A. Bonami and S. Grellier, "Hankel operators and weak factorization for Hardy-Orlicz spaces," Colloquium Mathematicum, vol. 118, no. 1, pp. 107-132, 2010.

[13] K. L. Avetisyan, "Weighted spaces of harmonic and holomorphic functions," Armenian Journal of Mathematics, vol. 2, no. 4, 2009.

[14] J. Gonessa and K. Zhu, "A sharp norm estimate for weighted Bergman projections on the minimal ball," New York Journal of Mathematics, vol. 17a, pp. 101-112, 2011.

[15] G. Mengotti and E. H. Youssfi, "The weighted Bergman projection and related theory on the minimal ball," Bulletin des Sciences Mathématiques, vol. 123, no. 7, pp. 501-525, 1999.

[16] K. T. Hahn and P. Pflug, "On a minimal complex norm that extends the real Euclidean norm," Monatshefte für Mathematik, vol. 105, no. 2, pp. 107-112, 1988.

[17] K. Oeljeklaus and E. H. Youssfi, "Proper holomorphic mappings and related automorphism groups," The Journal of Geometric Analysis, vol. 7, no. 4, pp. 623-636, 1997.

[18] J. M. Ortega and J. Fàbrega, "Hardy's inequality and embeddings in holomorphic Triebel-Lizorkin spaces," Illinois Journal of Mathematics, vol. 43, no. 4, pp. 733-751, 1999. 


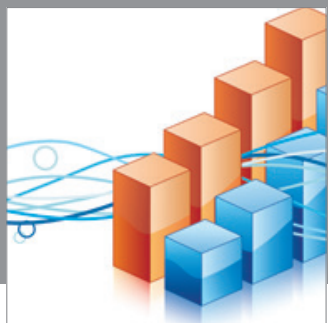

Advances in

Operations Research

mansans

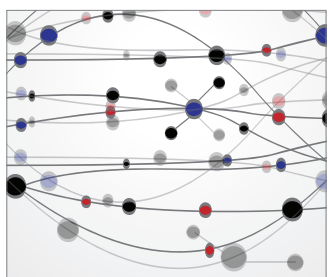

The Scientific World Journal
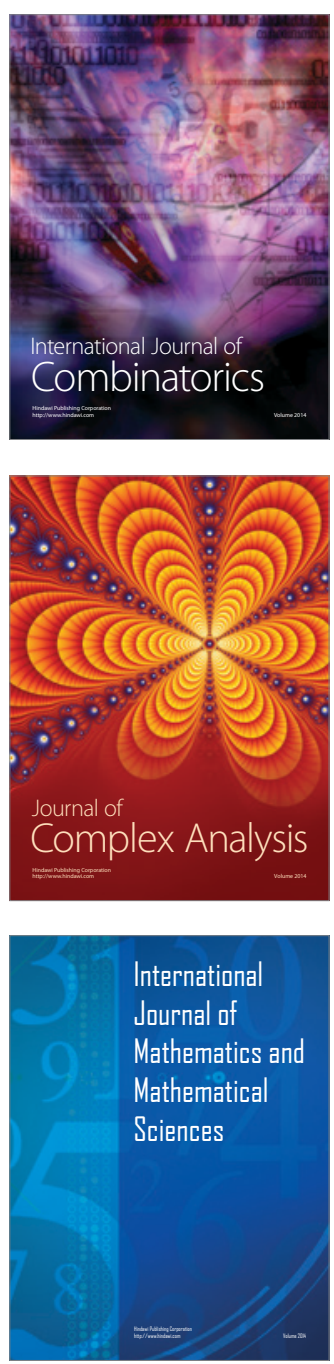
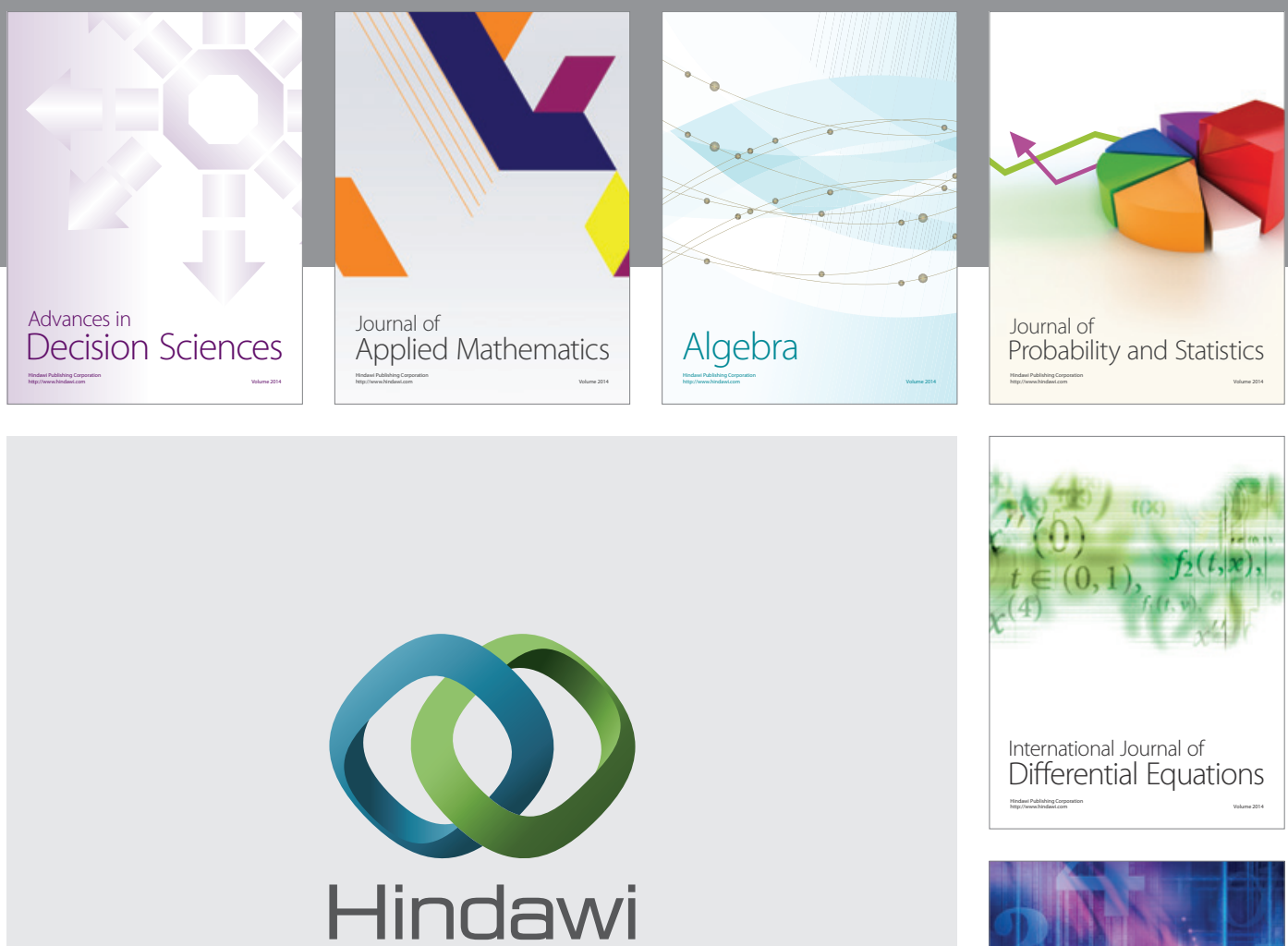

Submit your manuscripts at http://www.hindawi.com
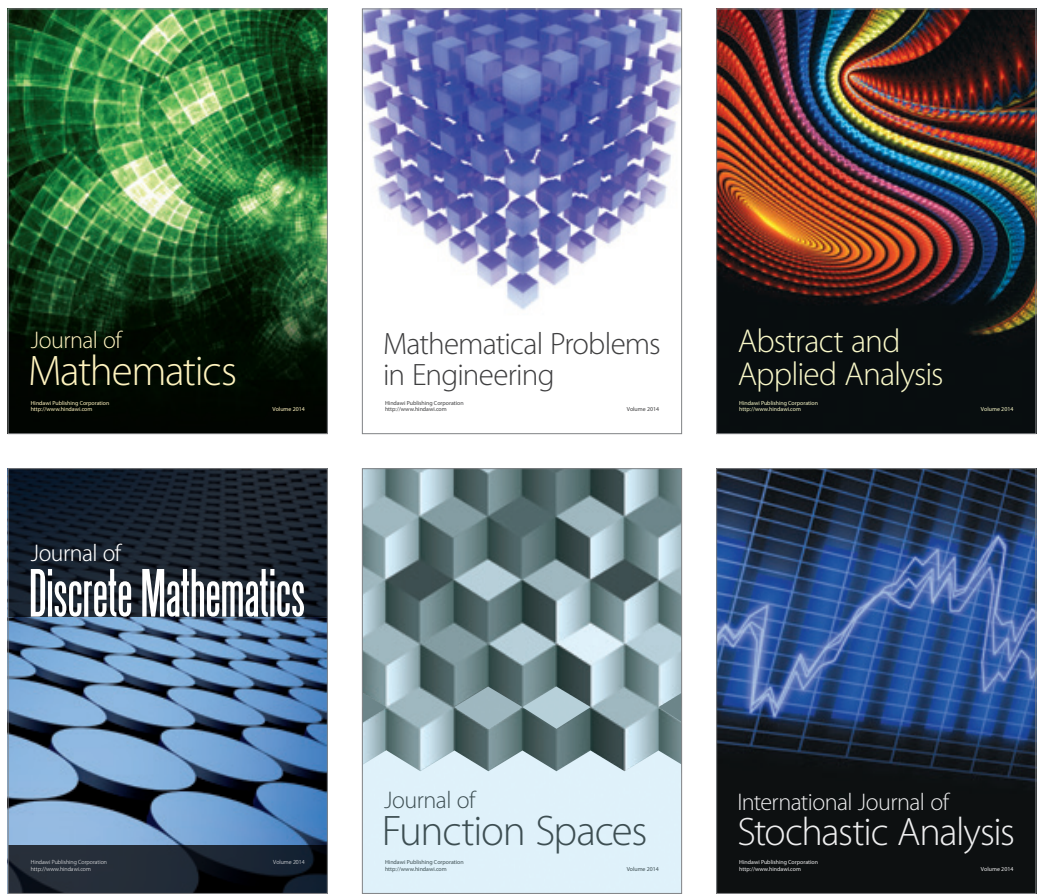

Journal of

Function Spaces

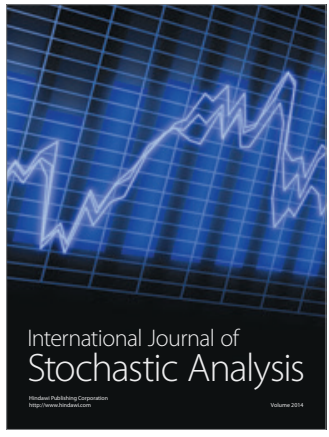

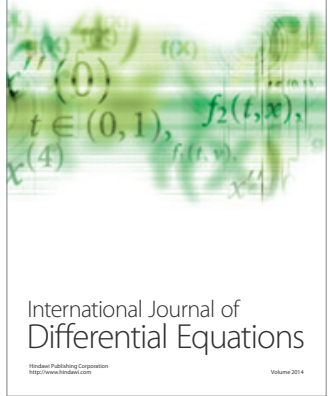
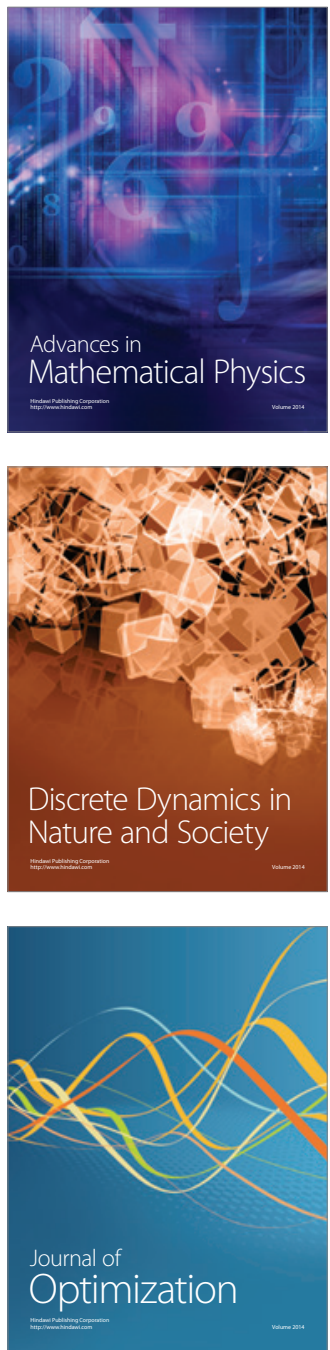